\title{
Excitatory Hindbrain-Forebrain Communication Is Required for Cisplatin-Induced Anorexia and Weight Loss
}

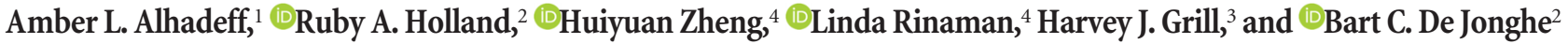 \\ ${ }^{1}$ Department of Biology, ${ }^{2}$ Department of Biobehavioral Health Sciences, and ${ }^{3}$ Department of Psychology, University of Pennsylvania, Philadelphia, \\ Pennsylvania 19104, and ${ }^{4}$ Department of Neuroscience, University of Pittsburgh, Pittsburgh, Pennsylvania 15260
}

Cisplatin chemotherapy is commonly used to treat cancer despite severe energy balance side effects. In rats, cisplatin activates nucleus tractus solitarius (NTS) projections to the lateral parabrachial nucleus (IPBN) and calcitonin-gene related peptide (CGRP) projections from the IPBN to the central nucleus of the amygdala $(\mathrm{CeA})$. We demonstrated previously that CeA glutamate receptor signaling mediates cisplatin-induced anorexia and body weight loss. Here, we used neuroanatomical tracing, immunofluorescence, and confocal imaging to demonstrate that virtually all NTS $\rightarrow$ IPBN and IPBN $\rightarrow$ CeA CGRP projections coexpress vesicular glutamate transporter 2 (VGLUT2), providing evidence that excitatory projections mediate cisplatin-induced energy balance dysregulation. To test whether IPBN $\rightarrow \mathrm{CeA}$ projection neurons are required for cisplatin-induced anorexia and weight loss, we inhibited these neurons chemogenetically using a retrograde Cre-recombinase-expressing canine adenovirus-2 in combination with Cre-dependent inhibitory Designer Receptors Exclusive Activated by Designer Drugs (DREADDs) before cisplatin treatment. Inhibition of IPBN $\rightarrow$ CeA neurons attenuated cisplatin-induced anorexia and body weight loss significantly. Using a similar approach, we additionally demonstrated that inhibition of NTS $\rightarrow$ IPBN neurons attenuated cisplatin-induced anorexia and body weight loss significantly. Together, our data support the view that excitatory hindbrain-forebrain projections are necessary for cisplatin's untoward effects on energy intake, elucidating a key neuroanatomical circuit driving pathological anorexia and weight loss that accompanies chemotherapy treatment.

Key words: amygdala; anorexia; chemotherapy; cisplatin; glutamate; hindbrain

Significance Statement

Chemotherapy treatments are commonly used to treat cancers despite accompanying anorexia and weight loss that may limit treatment adherence and reduce patient quality of life. Strikingly, we lack a neural understanding of, and effective treatments for, chemotherapy-induced anorexia and weight loss. The current data characterize the excitatory nature of neural projections activated by cisplatin in rats and reveal the necessity of specific hindbrain-forebrain projections for cisplatin-induced anorexia and weight loss. Together, these findings help to characterize the neural mechanisms mediating cisplatin-induced anorexia, advancing opportunities to develop better-tolerated chemotherapies and adjuvant therapies to prevent anorexia and concurrent nutritional deficiencies during cancer treatment.

\section{Introduction}

Over half a million cancer patients each year undergo chemotherapy in the United States and often suffer from severe side effects (e.g., nausea/vomiting, appetite loss, anorexia, and weight loss) that devastate quality of life and require constant management (Cohen et al.,

\footnotetext{
Received Aug. 27, 2016; revised Nov. 4, 2016; accepted Nov. 19, 2016.

Author contributions: A.L.A., H.Z., L.R., H.J.G., and B.C.D.J. designed research; A.L.A., R.A.H., H.Z., and B.C.D.J. performed research; A.L.A., H.Z., L.R., and B.C.D.J. analyzed data; A.L.A., L.R., H.J.G., and B.C.D.J. wrote the paper.

This work was supported by the University Research Foundation, University of Pennsylvania and the National Institutes of Health (Grants NIH-DK21397, NIH-NS08463, and NIH-MH59911).

The authors declare no competing financial interests.

Correspondence should be addressed to Bart C. De Jonghe, Department of Biobehavioral Health Sciences, University of Pennsylvania, 418 Curie Blvd., Philadelphia, PA 19104. E-mail: bartd@nursing.upenn.edu. DOI:10.1523/JNEUROSCI.2714-16.2016

Copyright $\odot 2017$ the authors $\quad 0270-6474 / 17 / 370362-09 \$ 15.00 / 0$
}

2007; Grunberg, 2012; Hattori et al., 2013; Hesketh et al., 2016; Jordan et al., 2015). Cisplatin, which is one of the oldest and most widely used and studied chemotherapies (Hesketh, 2008; Hesketh et al., 2016), induces these side effects in $>90 \%$ of patients (Hesketh, 2008 ), including appetite loss and anorexia in $\sim 50 \%$ of patients (Winton et al., 2005; Hong et al., 2006). Although the development of antiemetic pharmacotherapies over the past 25 years has improved control over chemotherapy-induced vomiting (Hattori et al., 2013; Jordan et al., 2015), antiemetic drugs do not control chemotherapy-induced appetite loss, anorexia, and marked loss of body weight sufficiently (Rudd and Andrews, 2005; Percie du Sert et al., 2011). This presents a significant problem for patients who are unable to maintain adequate nutrition in addition to reduced quality of life. Given the gravity of these chemotherapeutic side effects in such a large percentage of patients, improved understanding of the 
mechanisms and neural signaling pathways underlying these effects should promote the development of improved treatment options and is a clear priority for patient care.

There is accumulating evidence of a role for hindbrain-forebrain communication in cisplatin-induced anorexia and weight loss. Building upon studies demonstrating that cisplatin activates neurons in the nucleus tractus solitarius (NTS), lateral parabrachial nucleus (lPBN), and central nucleus of the amygdala (CeA) (Horn et al., 2007; De Jonghe and Horn, 2009; Horn, 2009; Alhadeff et al., 2015), our recent work suggests that projections between these regions may mediate cisplatin-induced anorexia and body weight loss, likely via CGRP/glutamatergic signaling (Alhadeff et al., 2015). However, the direct functional role of these projections in cisplatin-induced anorexia and body weight loss remained to be determined.

In the present study, immunohistochemistry and confocal imaging were used to reveal the glutamatergic nature of $\mathrm{NTS} \rightarrow \mathrm{IPBN}$ and $\mathrm{IPBN} \rightarrow$ CeA projections and projection-specific Designer Receptors Exclusively Activated by Designer Drugs (DREADD)-mediated neuronal inhibition was used to demonstrate the necessity of these excitatory projections in mediating cisplatin-induced side effects. Our data indicate that glutamatergic NTS $\rightarrow 1 \mathrm{PBN}$ and $\mathrm{IPBN} \rightarrow \mathrm{CeA}$ projections are required for cisplatin-induced anorexia and weight loss.

\section{Materials and Methods}

Immunohistochemical analysis of $N T S \rightarrow I P B N$ and $l P B N \rightarrow C e A$ projection phenotypes

Subjects and drugs. Male Sprague-Dawley rats (250-300 g upon arrival; Harlan Laboratories) were individually housed in hanging metal cages in a $12 \mathrm{~h}$ light $/ 2 \mathrm{~h}$ dark cycle with ad libitum access to pelleted chow (Purina Rodent Chow 5001) and water. All procedures were reviewed and approved by the Institutional Animal Care and Use Committee at the University of Pittsburgh.

Biotinylated dextran amine (BDA, D-1956, $10 \mathrm{~K} \mathrm{MW;} \mathrm{Invitrogen)} \mathrm{was}$ diluted to $5 \%$ solution in sterile saline for microinjection.

Surgery. Rats were anesthetized by isofluorane inhalation $(1-3 \%$ in oxygen; Halocarbon Laboratories). The skin over the dorsal neck surface was shaved, sterilized, incised along the midline, and the neck muscles retracted to expose the atlantooccipital membrane overlying the dorsal surface of the caudal medulla. With the aid of a surgical microscope, the membrane was opened with a sterile needle to reveal obex on the dorsomedial medullary surface at the caudal tip of the AP. To target tracer delivery into the caudal medial NTS, a glass iontophoretic micropipette (inner tip diameter $\sim 20 \mu \mathrm{m}$ ) filled with BDA was positioned $0.4 \mathrm{~mm}$ lateral to obex and then the tip was advanced $0.4 \mathrm{~mm}$ below the medullary surface at a $10^{\circ}$ angle from the vertical plane. BDA was delivered using a $7 \mathrm{~s}$ on/off pulsed current of $5 \mu \mathrm{A}$ for $10 \mathrm{~min}$. The glass pipette was removed 2 min after tracer delivery and the skin incision was sutured. A representative image of a BDA tracer infusion site is provided in Figure 2A.

Perfusion fixation and tissue preparation. Ten days after iontophoretic delivery of BDA into the caudal medial NTS or after 1 week of laboratory acclimation (for non-tracer-injected cases), rats were deeply anesthetized with a lethal dose of pentobarbital sodium (Fatal Plus; $100 \mathrm{mg} / \mathrm{kg}$ BW, IP; Butler Schein) and transcardially perfused, first with $100 \mathrm{ml}$ of $0.15 \mathrm{M} \mathrm{NaCl}$ and then with $400 \mathrm{ml}$ of $4 \%$ paraformaldehyde containing L-lysine and sodium metaperiodate (McLean and Nakane, 1974) in $0.1 \mathrm{M}$ phosphate buffer, $\mathrm{pH}$ 7.4. Fixed brains were removed from the skull, blocked, postfixed for $5-6 \mathrm{~h}$ at $4^{\circ} \mathrm{C}$, submerged in $20 \%$ sucrose solution for $18-24 \mathrm{~h}$ at $4^{\circ} \mathrm{C}$, and then sectioned in the coronal plane from the upper cervical spinal cord through the rostral corpus callosum using a freezing stage sliding microtome (Leica). Six series of floating sections (35 $\mu \mathrm{m}$ thickness) were collected into buffer. Tissue sections were either immediately processed for immunohistochemistry or stored at $-20^{\circ} \mathrm{C}$ in cryopreservant $(1.0 \mathrm{M}$ sucrose, $30.0 \%$ ethylene glycol, and
$1.0 \%$ polyvinylpyrrolidone- 40 in $50.0 \mathrm{~mm}$ sodium phosphate buffer, $\mathrm{pH}$ 7.4) for later use.

Dual immunofluorescence labeling for CGRP and VGLUT2. Antibodies were diluted in $0.1 \mathrm{M}$ phosphate buffer $(\mathrm{PB})$ containing $0.3 \%$ Triton $\mathrm{X}-100$ and $1.0 \%$ donkey serum. Tissue sections were rinsed, pretreated for $20 \mathrm{~min}$ in $1.0 \%$ sodium borohydride, rinsed, incubated for $15 \mathrm{~min}$ in $0.15 \%$ hydrogen peroxide, rinsed, and then incubated for $44 \mathrm{~h}$ in a primary antibody mixture containing guinea pig anti-CGRP (1:1.5K, Peninsula Lab, T-5053; RRID: AB_1113068) and mouse anti-VGLUT2 (1: 1K, Millipore, MAB5504; RRID: AB_2187552). Sections were rinsed and incubated $2 \mathrm{~h}$ at room temperature in a secondary antibody mixture containing Alexa Fluor 647-conjugated donkey anti-mouse IgG (to reveal VGLUT2 immunolabeling) combined with HRP-conjugated donkey anti-guinea pig IgG (1:400 each, Jackson ImmunoResearch). After $\mathrm{PB}$ rinses, the VGLUT2-labeled sections were reacted for $10 \mathrm{~min}$ with tyramide conjugated to Cy3 (1:300 in amplification diluent; Perkin Elmer) to reveal CGRP labeling. Reacted sections were rinsed and mounted onto Superfrost Plus Microscope Slides, allowed to dry, dehydrated, defatted in a series of graded ethanols followed by xylene, and coverslipped with Cytoseal 60 mounting medium.

Triple immunofluorescence labeling for BDA, VGLUT2, and CGRP. Triple-labeling procedures were performed sequentially at room temperature. After pretreatment as above, tissue sections were incubated for $22 \mathrm{~h}$ in guinea pig anti-VGLUT2 (1:1.5 K; Millipore, AB2251; RRID: AB_1587626), rinsed, and then incubated in Alexa Fluor 647-conjugated donkey anti-guinea pig IgG (to reveal VGLUT2 immunolabeling). Thirty minutes after the onset of this incubation, HRP-conjugated streptavidin ( $1: 8 \mathrm{~K}$; Jackson Laboratories) was added directly to the same solution and tissue sections were incubated for an additional $2 \mathrm{~h}$. After rinsing, sections were reacted for $10 \mathrm{~min}$ with tyramide conjugated to Cy3 (1:400 in amplification diluent; PerkinElmer) to reveal BDA labeling. After rinsing, tissues were treated for $15 \mathrm{~min}$ in $0.15 \%$ hydrogen peroxide, rinsed, incubated for $43 \mathrm{~h}$ in rabbit anti-CGRP (1:5K, provided by Dr. Catia Sternini, UCLA; RRID: AB_2314163), rinsed, and incubated in HRPconjugated donkey anti-rabbit IgG (1:500; Millipore AP128P). CGRP was revealed by $10 \mathrm{~min}$ reaction with tyramide plus conjugated to FITC (1:400 in amplification diluent; PerkinElmer). Reacted sections were rinsed and mounted and covered as mentioned above.

Primary antibody specificity. Of the four primary antibodies used in this work, three (mouse anti-VGLUT2, guinea pig anti-VGLUT2, and rabbit anti-CGRP) are included in the Journal of Comparative Neurology antibody database (V.14), which provides citations documenting antibody specificity in rat brain. To confirm specificity of the fourth antibody (guinea pig anti-CGRP), we performed dual fluorescent immunolabeling in rat brain sections using that antibody together with the rabbit anti-CGRP antibody, followed by species-specific secondary antibodies conjugated to $\mathrm{Cy} 2$ and $\mathrm{Cy} 3$. The resulting fluorescent immunolabeling was completely colocalized, providing indirect evidence for specificity of the guinea pig anti-CGRP antibody.

Imaging and analysis of CGRP/VGLUT2 colocalization. Images were acquired with a Leica TCS SP8 confocal microscope using a $100 \times$ oilimmersion objective with $3.0 \times$ digital zoom. Images of Cy3 (CGRP) and Alexa Fluor 647 (VGLUT2) axonal labeling within the CeA were collected sequentially with a $552 \mathrm{~nm}$ OPSL laser and a $638 \mathrm{~nm}$ diode laser, respectively, to avoid potential signal contamination. Analysis of CGRP and VGLUT2 colocalization within the same pixel (pixel size $=0.074$ $\left.\mu \mathrm{m}^{2}\right)$ was conducted on 32 optical slices $(0.28 \mu \mathrm{m}$ thickness $)$ within the $z$-stack. The extent of within-pixel fluorescent signal colocalization was quantified using the "colocalization threshold" measure in ImageJ (2.0.0), in which a detection threshold for each channel is determined automatically to avoid subjective bias. Colocalization was calculated in each optical slice and then collapsed into a maximal $z$-projection to reveal colocalized pixels across the entire image thickness.

Imaging and analysis of BDA/VGLUT2/CGRP triple labeling. Images were acquired as described above. Labeling of FITC (CGRP) and Alexa Fluor 647 (VGLUT2) within the IPBN and CeA were collected simultaneously with 488 and $638 \mathrm{~nm}$ lasers, respectively. Images of Alexa Fluor Cy3 (BDA) labeling were collected sequentially using a 552 laser to avoid potential signal contamination. In one representative case with an ideal 
caudal NTS tracer injection site, analysis of 1PBN BDA and VGLUT2 colocalization within the same pixel (pixel size $=0.072 \mu \mathrm{m}^{2}$ ) was conducted on 57 optical slices $(0.39 \mu \mathrm{m}$ thickness) within the $z$-stack. The extent of within-pixel fluorescent signal colocalization was quantified as described above.

\section{Chemogenetic behavioral experiments}

Subjects and drugs. Male Sprague-Dawley rats (250-265 g upon arrival; Charles River Laboratories) were housed individually in hanging metal cages in a $12 \mathrm{~h}$ light $/ 12 \mathrm{~h}$ dark cycle. Rats had ad libitum access to pelleted chow (Purina Rodent Chow 5001) and water except when otherwise noted. All procedures conformed to and received approval from the institutional standards of the University of Pennsylvania Institutional Animal Care and Use Committee.

Cisplatin (cis-diamineplatinum dichloride; Sigma-Aldrich) was administered at a dose of $6 \mathrm{mg} / \mathrm{kg}$ in $0.15 \mathrm{M} \mathrm{NaCl}$. The cisplatin solution was sonicated and vortexed immediately before injection. Clozapine-Noxide (CNO; Tocris Bioscience) was administered at a dose of $1 \mathrm{mg} / \mathrm{kg}$ in saline.

Surgery. Rats received intramuscular anesthesia [ketamine $(90 \mathrm{mg} / \mathrm{kg}$; Butler Animal Health Supply), xylazine (2.7 mg/kg; Anased), and acepromazine $(0.64 \mathrm{mg} / \mathrm{kg}$; Butler Animal Health Supply) and subcutaneous analgesia $(2.0 \mathrm{mg} / \mathrm{kg}$ Metacam; Boehringer Ingelheim Vetmedica) for all surgeries.

Rats were injected bilaterally with the retrograde canine adenovirus- 2 (IGMM) delivered into either the CeA or lPBN. In the same surgical session, rats also were injected bilaterally with the inhibitory (Gicoupled) DREADD virus AAV-hSyn-DIO-hM ${ }_{4} \mathrm{D}$-mCherry or control virus AAV-hSyn-DIO-mCherry (UNC Vector Core, Chapel Hill, NC) delivered into the IPBN or caudal medial NTS. All viruses were diluted to a titer of $2 \times 10^{9}$ viral genomes $/ \mathrm{ml}$ and injected ( $500 \mathrm{nl} /$ hemisphere $)$ at a rate of $0.0031 \mathrm{ml} / \mathrm{min}$. The following stereotaxic coordinates were used for each injection site: CeA: $\pm 4.3 \mathrm{~mm}$ lateral from midline, $2.4 \mathrm{~mm}$ posterior from bregma, and $8.1 \mathrm{~mm}$ ventral from skull surface; $1 \mathrm{PBN}$ : $\pm 2.0 \mathrm{~mm}$ lateral from midline, $0.6 \mathrm{~mm}$ anterior to lambda, and $7.7 \mathrm{~mm}$ ventral from skull surface; NTS: $\pm 0.5 \mathrm{~mm}$ lateral from midline, $1.9 \mathrm{~mm}$ anterior to occipital, $8.8 \mathrm{~mm}$ ventral from skull surface. All rats were given 2-3 weeks for recovery from surgery and transgene expression (Carter et al., 2013; Boender et al., 2014). Brains were sectioned postmortem to verify injection placement. Rats in qhixh DREADD/mCherry viral injections extended beyond the $1 \mathrm{PBN}$ (8.7-9.7 $\mathrm{mm}$ posterior to bregma, according to Paxinos and Watson, 2005) or caudal medial NTS (13.8$14.2 \mathrm{~mm}$ posterior to bregma) were excluded from data analyses. One rat was excluded from behavioral analyses on this basis. Representative images of mCherry reporter expression after injection of the inhibitory DREADDs ( $\mathrm{hM}_{4} \mathrm{D}$-mCherry) into the $1 \mathrm{PBN}$ or caudal medial NTS are depicted in Figure 3, $A$ and $B$, respectively.

Experimental procedure. To examine the necessity of $\mathrm{IPBN} \rightarrow \mathrm{CeA}$ or NTS $\rightarrow$ PBN projections in cisplatin-induced anorexia and body weight loss, rats with inhibitory DREADD expression $(n=11$ and $n=13$ for $\mathrm{IPBN} \rightarrow \mathrm{CeA}$ or NTS $\rightarrow \mathrm{PBN}$ projection, respectively) or rats expressing the control virus $(n=10$ and $n=9$ for $1 \mathrm{PBN} \rightarrow$ CeA or NTS $\rightarrow$ IPBN projection, respectively) received intraperitoneal $\mathrm{CNO}$ or saline in a pseudo-counterbalanced manner as described previously (Alhadeff et al., 2015) immediately before and $24 \mathrm{~h}$ after intraperitoneal saline injection. The dose of CNO $(1 \mathrm{mg} / \mathrm{kg}$ ) was taken from the literature (Carter et al., 2013; Boender et al., 2014) and our preliminary studies (data not shown) indicating no independent effect of this CNO dose on food intake or body weight. Food intake and body weight were recorded at 24 and $48 \mathrm{~h}$ after injection. At least $72 \mathrm{~h}$ later, the same rats were injected with $\mathrm{CNO}$ or saline immediately before and $24 \mathrm{~h}$ after intraperitoneal cisplatin injection. The dose of cisplatin $(6 \mathrm{mg} / \mathrm{kg})$ was chosen based on its ability to reduce food intake and body weight (De Jonghe and Horn, 2008; Alhadeff et al., 2015). Again, food intake and body weight were recorded at 24 and $48 \mathrm{~h}$ after injection. Rats were killed $48 \mathrm{~h}$ after cisplatin injection and brains were analyzed for mCherry reporter expression.

\section{Statistical analyses}

All data are expressed as group mean \pm SEM. Two-way ANOVAs in a $2 \times$ 2 between-subjects design were performed to evaluate the main effects of experimental treatment group (i.e., IP CNO/saline vs IP cisplatin/saline) on food intake and body weight change. Planned post hoc comparisons were conducted with least significant difference (LSD) tests when applicable. Statistical differences between mean values were calculated using SAS version 9.2 software (SAS Institute).

\section{Results}

\section{$\mathrm{IPBN} \rightarrow \mathrm{CeA}$ and NTS $\rightarrow$ IPBN neurons are glutamatergic}

CGRP-positive fibers and terminals within the rat CeA are known to derive from the IPBN (Schwaber et al., 1988). Dual immunofluorescent colocalization of CGRP and VGLUT2 within the CeA is depicted in Figure 1. A conservative thresholdbased quantitative colocalization analysis of CGRP and VGLUT2 immunolabeling in axon varicosities located in the CeA (Fig. $1 B-D)$ revealed that $55.0 \%$ of pixels that were above threshold for CGRP immunofluorescence also were above threshold for VGLUT2 (Fig. 1E). Figure $1 D$ illustrates qualitatively that all visible CGRP-positive profiles also expressed VGLUT2 immunofluorescence. As expected, given the wider distribution and greater density of VGLUT2-positive versus CGRP-positive terminals within the CeA, $28.0 \%$ of pixels that were above threshold for VGLUT2 also were above threshold for CGRP. 3D images were digitally rotated to confirm extensive colocalization of VGLUT2 with CGRP immunofluorescence in the same varicose profiles within the CeA (Fig. 1B). Colocalization correlation coefficients are provided in Figure $1 E$; note that the correlation coefficients do not reflect the percentages of colocalization, but are the within-pixel correlation coefficients of signal intensity for each fluorophore. These data support the view that CGRP-positive IPBN neurons innervating the CeA are glutamatergic.

Colocalization of BDA-positive axons (anterogradely labeled from the NTS; Fig. 2A) and VGLUT2 in the IPBN is depicted in Figure 2. Quantitative colocalization analysis of BDA- and VGLUT2-positive profiles revealed that $75.0 \%$ of pixels that were above threshold for BDA labeling also were above threshold for VGLUT2 and $73.0 \%$ of pixels that were above threshold for VGLUT2 also were above threshold for BDA. Figure $2 E$ illustrates qualitatively that all visible BDA-positive profiles within the IPBN also were VGLUT2-positive. Epifluorescence (Fig. 2C) and confocal (Fig. 2D,E) images depict double or triple labeling for BDA, VGLUT2, and CGRP. Figure $2 E$ reveals the nearly complete colocalization of BDA and VGLUT2 within the IPBN and Figure 2, $C$ and $D$, reveal the close apposition of BDA-labeled and VGLUT2-positive axons and varicosities with CGRP-positive IPBN neurons. These close appositions suggest the presence of synaptic connections, although verification of synapses would require ultrastructural analysis. Colocalization correlation coefficients for BDA and VGLUT2 are provided in Figure $2 F$. As noted above, values are the within-pixel correlation coefficients of signal intensity for each fluorophore. These data support the view that axonal projections from the caudal NTS to the IPBN are glutamatergic and include inputs that closely appose CGRPpositive IPBN neurons. A similar analysis conducted using a different anterograde tracer (PhAL) injected into the caudal NTS produced similar results (data not shown), providing further evidence that NTS $\rightarrow$ IPBN fibers targeting CGRP neurons coexpress VGLUT2.

\section{Chemogenetic behavioral experiments}

Inhibition of $l P B N \rightarrow C e A$ neurons attenuates cisplatin-induced anorexia and weight loss

In rats expressing inhibitory DREADDs in $\mathrm{PBN} \rightarrow \mathrm{CeA}$ neurons, significant cisplatin $\times$ CNO interactions were observed on both 

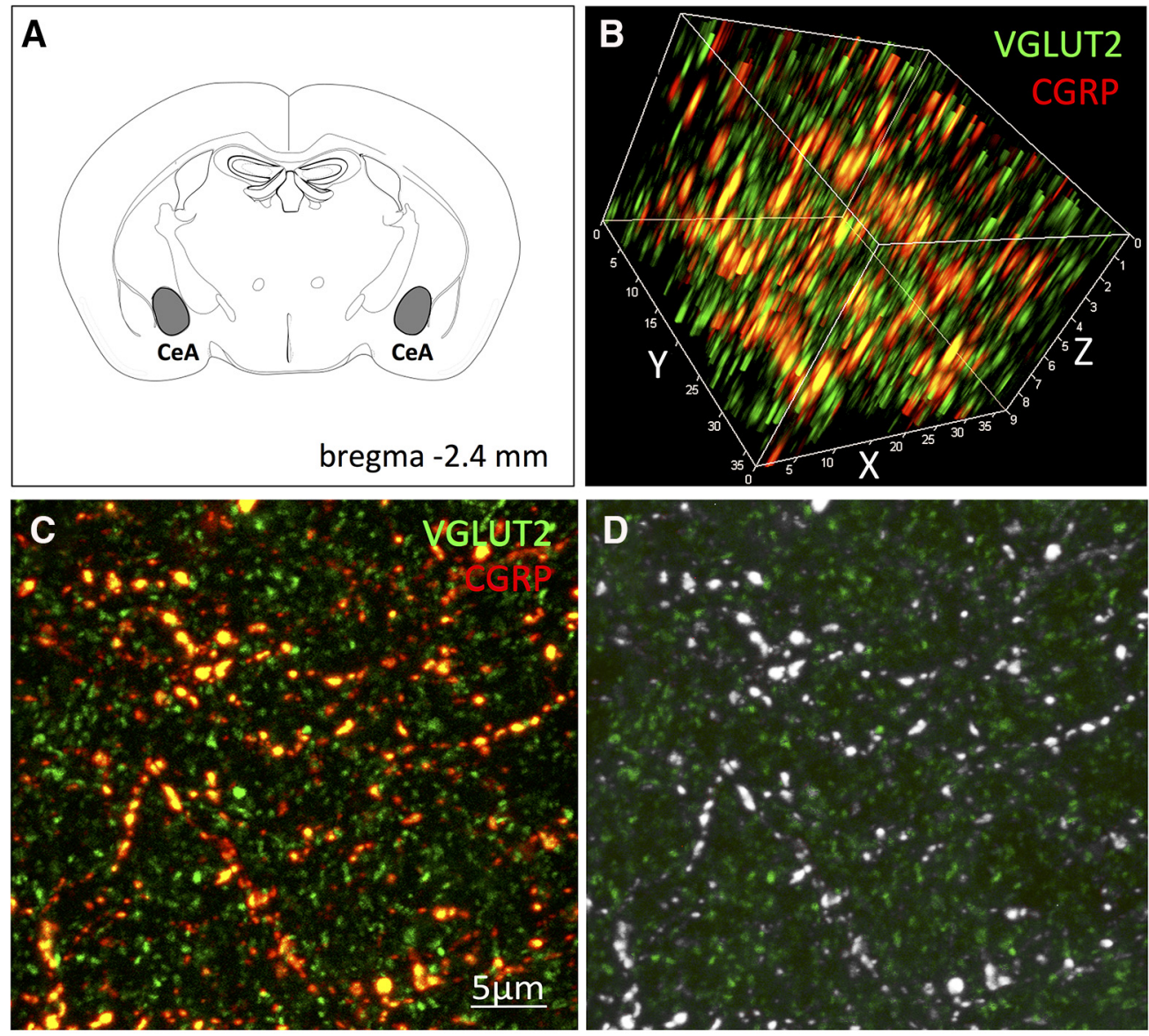

E \begin{tabular}{|c|c|c|c|}
\hline Rtotal & Rcoloc & tM-CGRP & tM-VGLUT2 \\
\hline 0.60 & 0.80 & 0.55 & 0.28 \\
\hline
\end{tabular}

Figure 1. Virtually all CGRP-positive fibers within the CeA coexpress VGLUT2. A, Location of (eA region analyzed. B, 3D rotated confocal z-stack image of VGLUT2 (green)- and CGRP (red)immunopositive axon terminals within the CeA. C, 2D flattened confocal image of (eA immunolabeling; orange/yellow profiles are double labeled for both VGLUT2 (green) and CGRP (red) immunofluorescence. $\boldsymbol{D}$, Confocal grayscale image of CeA immunolabeling: colocalized pixels (white) are those in which both VGLUT2 and CGRP signals are above their respective thresholds. Because all CGRP-positive profiles within the field also are VGLUT2 positive, they appear gray/white. E, Table of Pearson's and Mander's correlation coefficients for colocalization of CGRP and VGLUT2. Rtotal, Pearson's correlation coefficient for all pixels above background (no thresholds applied); Rcoloc, Pearson's correlation coefficient for all pixels in which both CGRP and VGLUT2 channels are above their respective thresholds; $T M$-CGRP, IM-VGLUT2, Mander's split channel colocalization coefficient using threshold.

food intake (Fig. 4B) and body weight change (Fig. 4C) (each $\left.F_{(1,18)} \geq 17.9 ; p<0.05\right)$. Post hoc analyses revealed that $\mathrm{CNO}$ treatment blocked the food intake-inhibitory effect of cisplatin at $24 \mathrm{~h}$ and attenuated cisplatin-induce anorexia and weight loss significantly $48 \mathrm{~h}$ after cisplatin injection. As expected, in rats injected with control AAV, the significant main effects of cisplatin treatment to suppress food intake and increase body weight loss at 24 and $48 \mathrm{~h}\left(\right.$ each $\left.F_{(1,18)} \geq 22.3 ; p<0.05\right)$ were not affected by CNO treatment (Fig. $4 E, F)$.

Inhibition of NTS $\rightarrow$ IPBN neurons attenuates cisplatin-induced anorexia and weight loss.

In rats expressing inhibitory DREADDs in NTS $\rightarrow$ IPBN neurons, a significant cisplatin $\times \mathrm{CNO}$ interaction on chow intake was observed at $48 \mathrm{~h}\left(F_{(1,22)}=8.9 ; p<0.05\right)$, with $\mathrm{CNO}$ attenuating cisplatin-induced anorexia ( $p<0.05$; Fig. $5 B)$. At 24 h, a nonsignificant trend for $\mathrm{CNO}$ to reduce cisplatin-induced anorexia was noted ( $p=0.10$; Fig. $5 B$ ). A significant cisplatin $\times \mathrm{CNO}$ interaction on body weight change was observed at both the 24 and $48 \mathrm{~h}$ time points (each $F_{(1,22)} \geq 24.2 ; p<0.05$; Fig. $5 C$ ). Post hoc analyses revealed that $\mathrm{CNO}$ injection blocked cisplatin-induced weight loss at $24 \mathrm{~h}$ and attenuated weight loss significantly at $48 \mathrm{~h}$. As expected, in rats injected with control AAV, cisplatin treatment suppressed food intake and increased body weight loss significantly at 24 and $48 \mathrm{~h}$ (each $\left.F_{(1,16)} \geq 14.5 ; p<0.05\right)$ and these effects were not altered by $\mathrm{CNO}$ treatment (Fig. $5 E, F$ ).

\section{Discussion}

Efforts to improve appetite and increase food intake in patients receiving chemotherapy will be most successful if they are based on a clear understanding of the underlying neural mechanisms. The current study investigated the neurochemical phenotype and functional significance of hindbrain-forebrain neuronal populations mediating cisplatin-induced anorexia and weight loss in an experimental rat model. Building upon previous findings suggesting that glutamate receptor signaling from CeA-projecting IPBN CGRP neurons triggers anorexia (Carter et al., 2013; Alhadeff et al., 2015), we demonstrate that $\mathrm{PBN} \rightarrow \mathrm{CeA}$ neurons expressing CGRP are glutamatergic and play a role in mediating cisplatin-induced anorexia. Chemogenetic inhibition of this excitatory projection attenuated cisplatin-induced anorexia and 

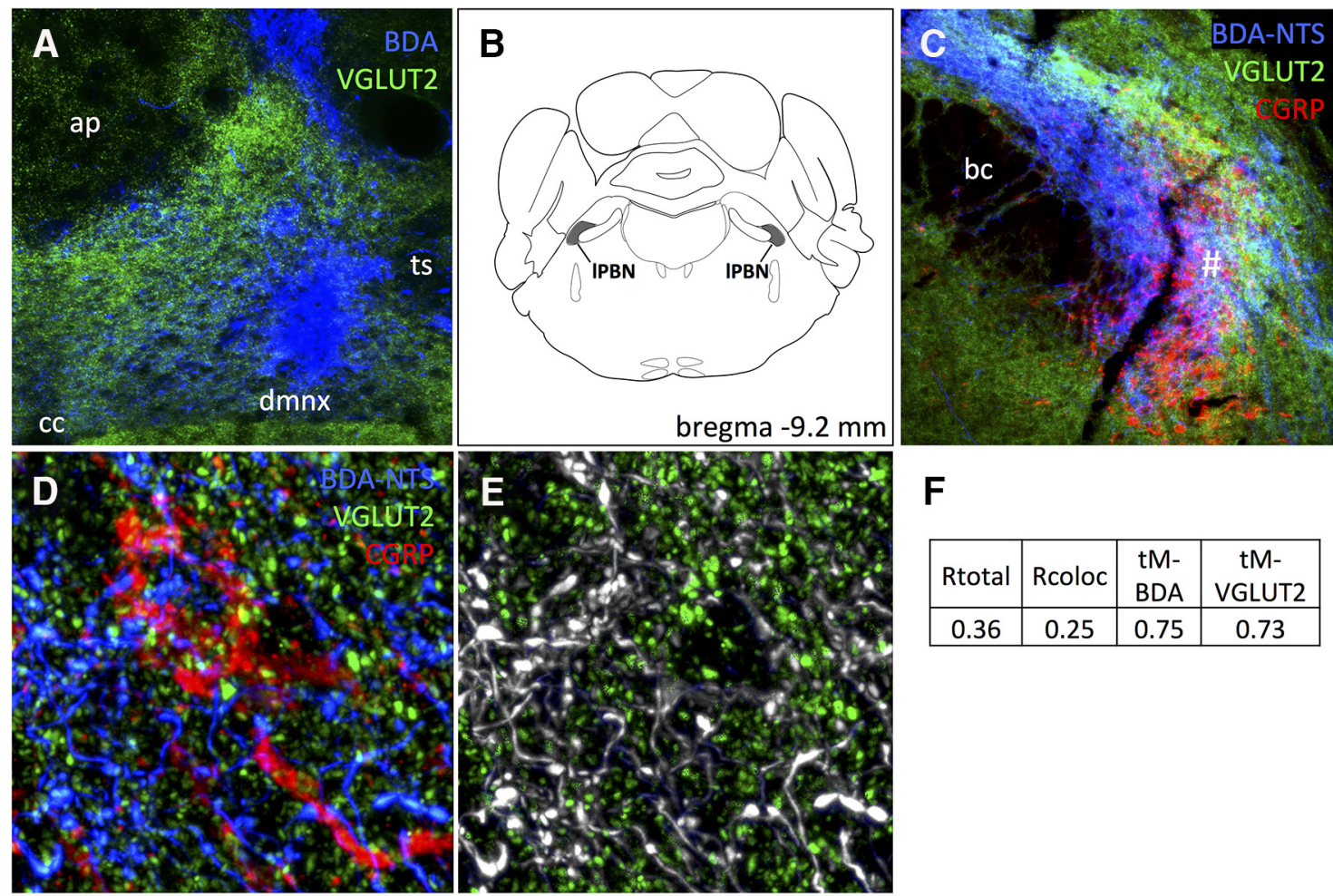

$F$

\begin{tabular}{|c|c|c|c|}
\hline Rtotal & Rcoloc & $\begin{array}{c}\text { tM- } \\
\text { BDA }\end{array}$ & $\begin{array}{c}\text { tM- } \\
\text { VGLUT2 }\end{array}$ \\
\hline 0.36 & 0.25 & 0.75 & 0.73 \\
\hline
\end{tabular}

Figure 2. Virtually all NTS $\rightarrow$ PBN fiber terminals in close apposition to IPBN GGRP neurons coexpress VGLUT2. A, Image of caudal NTS indicating BDA tracer injection placement (blue), with VGLUT2 (green) also imaged to reveal tissue structure and landmarks. ap, Area postrema; cc, central canal; dmnx, dorsal motor nucleus of the vagus; ts, solitary tract. $\boldsymbol{B}$, Location of IPBN region analyzed. C, Epifluorescence micrograph showing triple labeling of BDA + axons (blue), VGLUT2 (green), and (GRP + neurons (red) within the IPBN; $20 \times$ objective. bc, Brachium conjunctivum. \#Region of the IPBN analyzed for triple labeling. D, Higher-magnification confocal image showing triple labeling of BDA + axons and terminals (blue), VGLUT2 (green), and (GRP (red) within the IPBN; $100 \times$ oil objective and $3 \times$ digital zoom. $\boldsymbol{E}$, Confocal grayscale image: colocalized pixels (white) are those in which both BDA and VGLUT2 signals are above their respective thresholds. Because all BDA-positive profiles within the field also are VGLUT2 positive, they appear gray/white. $\boldsymbol{F}$, Table of Pearson's and Mander's correlation coefficients for colocalization of BDA and VGLUT2. Rtotal, Pearson's correlation coefficient for all pixels above background (no thresholds applied); Rcoloc, Pearson's correlation coefficient for all pixels in which both BDA and VGLUT2 channels are above their respective threshold; $T M-B D A$, $t M-V G L U T 2$, Mander's split channel colocalization coefficient using threshold.
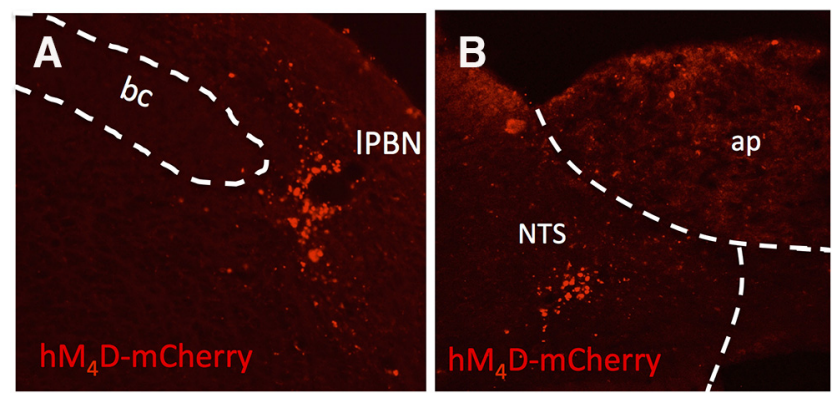

Figure 3. Representative images of inhibitory DREADD (hM4D-mCherry) virus expression within the IPBN ( $\boldsymbol{A})$ and caudal medial NTS $(\boldsymbol{B})$. ap, Area postrema; bc, brachium conjunctivum.

body weight loss. We additionally provide evidence that virtually all NTS $\rightarrow$ PBN projections that closely appose IPBN CGRP neurons express VGLUT2 and that inhibition of these neurons attenuates or blocks cisplatin-induced anorexia and weight loss. Together, these results demonstrate the necessity of excitatory NTS $\rightarrow$ IPBN and $\mathrm{IPBN} \rightarrow$ CeA projections for cisplatin-induced energy balance dysregulation.

Previous studies have demonstrated the importance of $1 \mathrm{PBN} \rightarrow$ CeA neurons in mediating anorexia (Carter et al., 2013; Campos et al., 2016). We reported recently that cisplatin activates IPBN CGRP neurons that project to the CeA and that glutamate receptor signaling within the $\mathrm{CeA}$ is required for cisplatininduced malaise and energy balance dysregulation (Alhadeff et al., 2015). It has been suggested that glutamate signaling mediates the anorexia resulting from activation of CGRP neurons within the IPBN (Carter et al., 2013). Here, we provide data from immunohistochemistry and confocal imaging to show conclusively that virtually all CGRP terminals within the CeA coexpress VGLUT2, a phenotypic marker for glutamatergic neurons. Given that the IPBN is the sole source of CGRP to the CeA in rats (Schwaber et al., 1988), these new data confirm the glutamatergic/excitatory nature of CGRP neural projections from $1 \mathrm{PBN} \rightarrow$ CeA.

To test directly the necessity of $\mathrm{PBNN} \rightarrow \mathrm{CeA}$ neurons in cisplatininduced anorexia and weight loss, we used a chemogenetic approach combining a Cre-recombinase-expressing retrograde virus (CAV-2Cre) with inhibitory (Gi-coupled) AAV-DREADDs (Armbruster et al., 2007; Carter et al., 2013; Boender et al., 2014). Specifically, we infused CAV-2-Cre into the CeA, followed by an infusion of the inhibitory DREADD into the IPBN, allowing for selective inhibition of $\mathrm{IPBN} \rightarrow \mathrm{CeA}$ neurons upon injection of the DREADD ligand CNO. Behavioral experiments demonstrated that chemogenetic inhibition of IPBN $\rightarrow$ CeA neurons attenuated cisplatin-induced anorexia and body weight loss significantly. In addition, animals injected with a control AAV for expression in IPBN $\rightarrow$ CeA projections displayed no effect of $\mathrm{CNO}$ on cisplatin-induced anorexia and weight loss. Together, these behavioral findings are consistent with results from previous studies reporting that $\mathrm{IPBN} \rightarrow$ CeA CGRP neurons are required for both anorexia and conditioned taste avoidance in mice after treatment with other noxious agents (i.e., lithium chloride and lipopolysaccharide; Carter et al., 2013; Carter et al., 2015). 


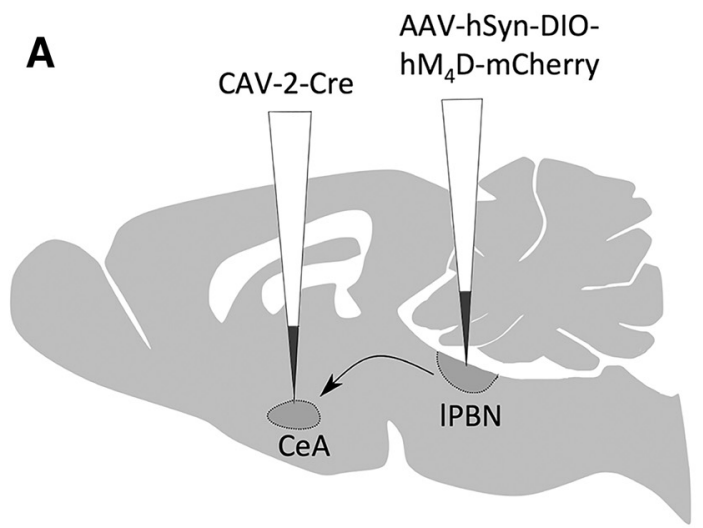

saline/saline

\section{$\mathrm{CNO} /$ saline}

B

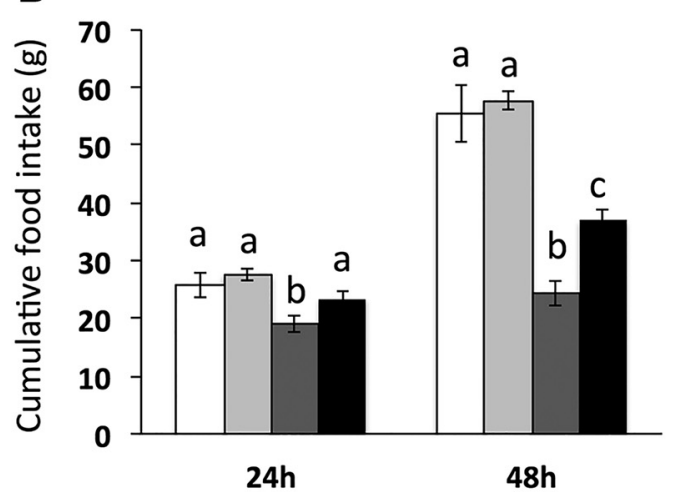

C

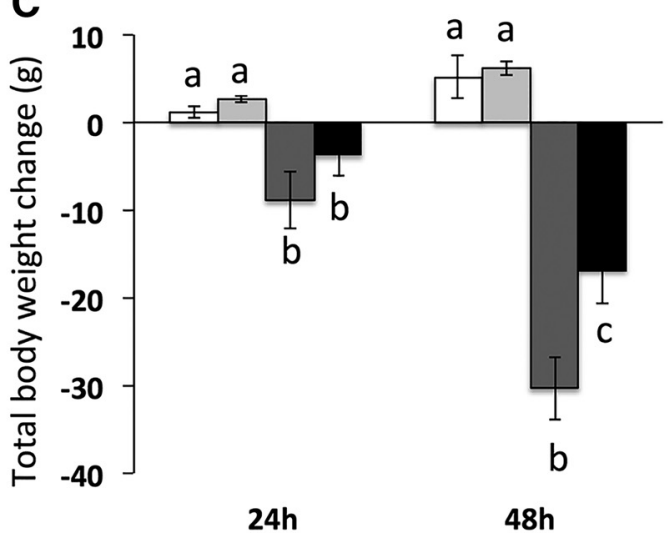

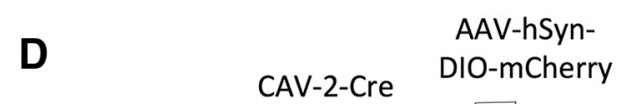

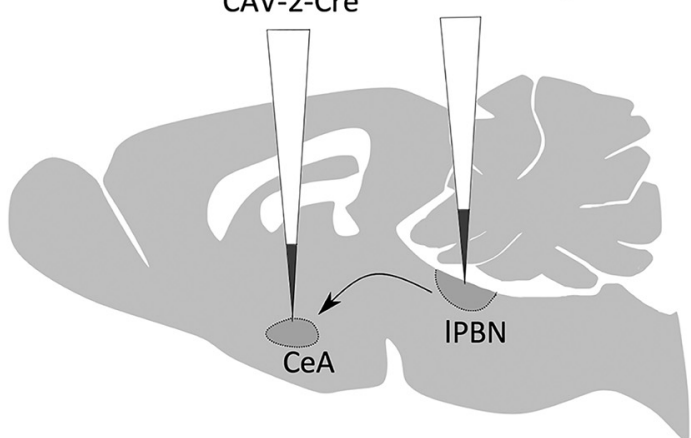

saline/cisplatin

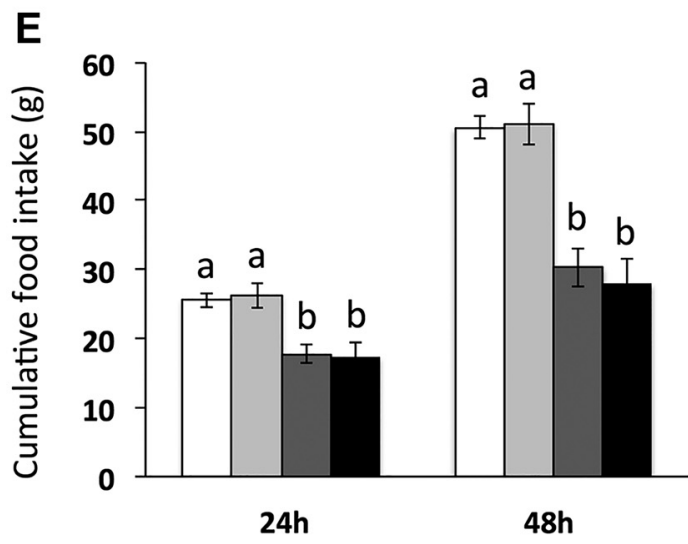

F

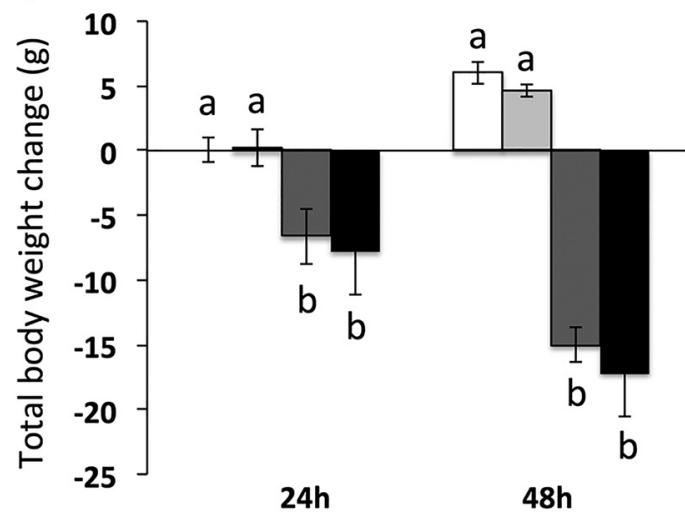

Figure 4. $\quad I P B N \rightarrow C$ CA projections are required for cisplatin-induced anorexia and weight loss. $A$, Surgical setup for IPBN $\rightarrow$ CeA neuron inhibition: bilateral injection of CAV-2-Cre in the CeA and the inhibitory Cre-dependent DREADD hM h $_{4}$ in the IPBN. $\boldsymbol{B}$, IPBN $\rightarrow$ CeA neuron inhibition by CNO attenuated cisplatin-induced anorexia. $\boldsymbol{C}$, IPBN $\rightarrow$ CeA neuron inhibition by CNO attenuated cisplatin-induced weight loss. $\boldsymbol{D}$, Surgical setup for IPBN $\rightarrow$ CeA experiment in control rats: bilateral injection of CAV-2-Cre in the CeA and AAV-mCherry in the IPBN. $\boldsymbol{E}$, CNO injection in control rats did not alter cisplatin-induced anorexia significantly. $\boldsymbol{F}$, CNO injection in control rats did not alter cisplatin-induced weight loss significantly. Data are expressed as mean \pm SEM; different letters denote significant differences between groups within time points ( $p<0.05, \boldsymbol{B}$ and $\boldsymbol{C}: n=5-6 /$ group, $\boldsymbol{E}$ and $\boldsymbol{F}: n=5 /$ group).

Interestingly, this same neural population has been implicated recently in meal size control and satiation (Campos et al., 2016). Collectively, it appears that $\mathrm{PBN} \rightarrow \mathrm{CeA}$ neurons are critically involved in the control of food intake such that physiological levels of activation lead to meal termination (Campos et al., 2016), whereas supraphysiological levels of activation such as those caused by illness (Carter et al., 2013; Carter et al., 2015) or, in the current case, systemic injection of toxin such as cisplatin, lead to pathophysiologic anorexia. Based on the aforementioned studies and our previous work (Alhadeff et al., 2015), we predict that IPBN $\rightarrow$ CeA CGRP neurons in particular drive cisplatin-induced anorexia and weight loss, but this must be confirmed directly in future studies.

Given that cisplatin also activates NTS $\rightarrow$ IPBN projections in rats (Alhadeff et al., 2015), we sought to phenotype this projection pathway. Using anterograde tracing, immunohistochemistry, and confocal imaging, we discovered that virtually all $\mathrm{NTS} \rightarrow$ IPBN fiber terminals in close apposition to IPBN CGRP neurons coexpress VGLUT2, evidence that this projection path- 

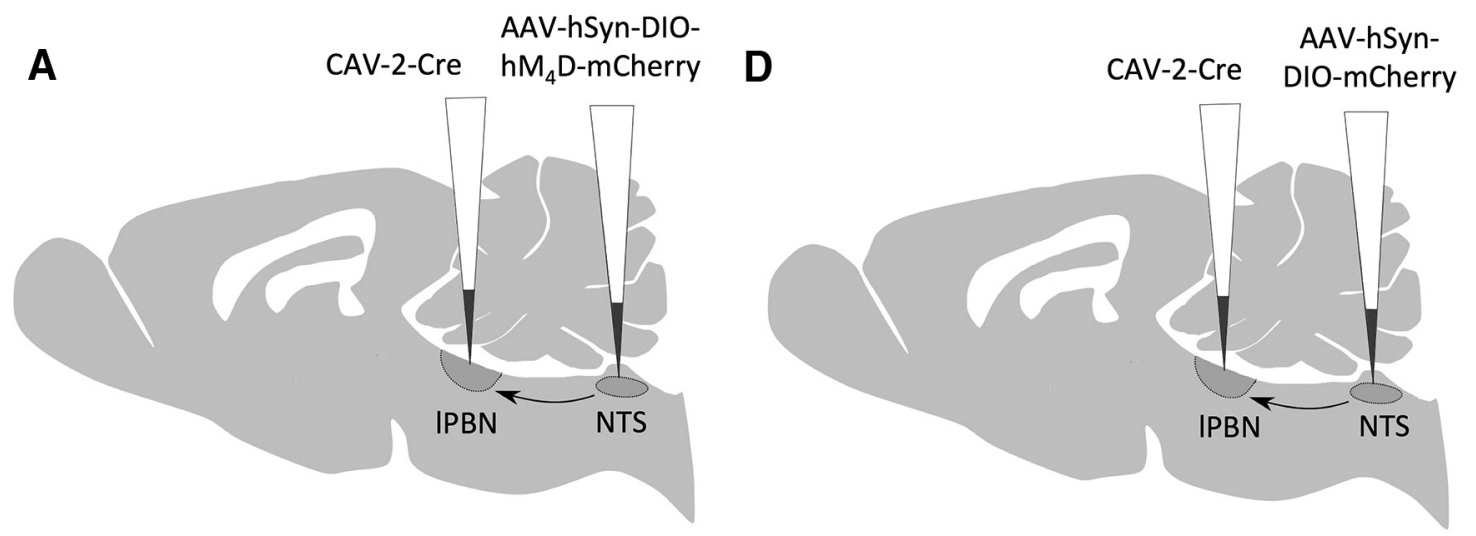

saline/saline

$\mathrm{CNO} /$ saline
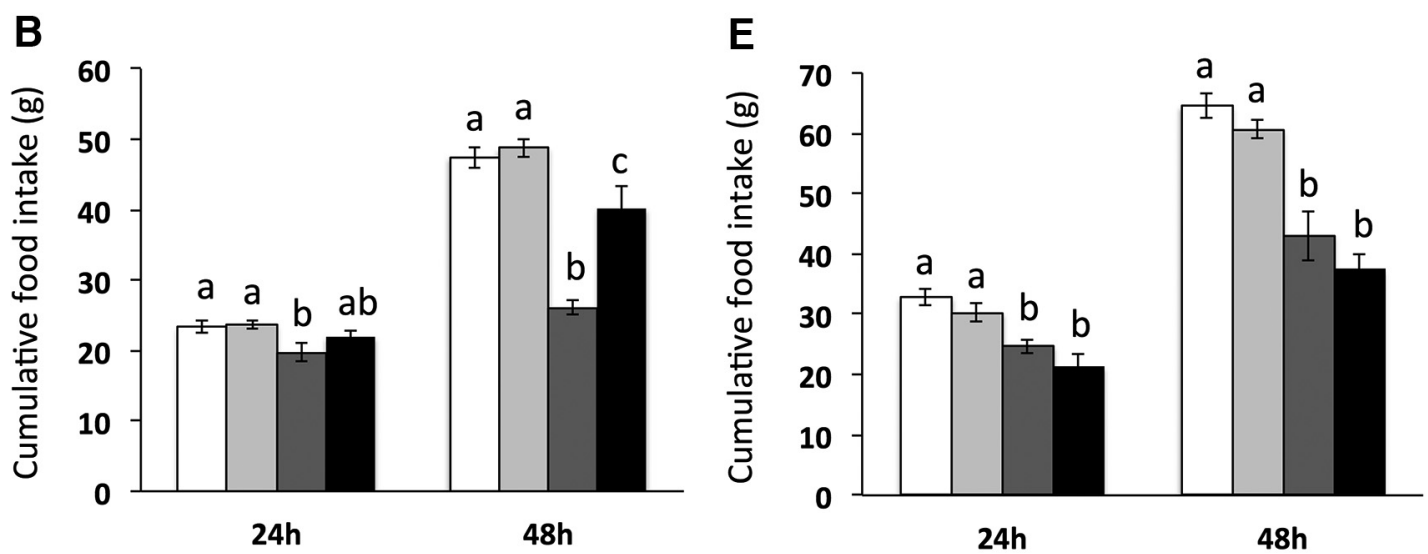

C

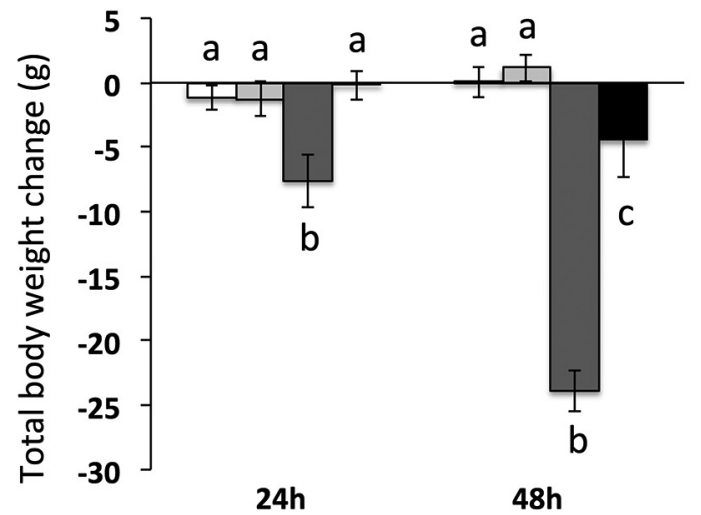

$\mathbf{F}$

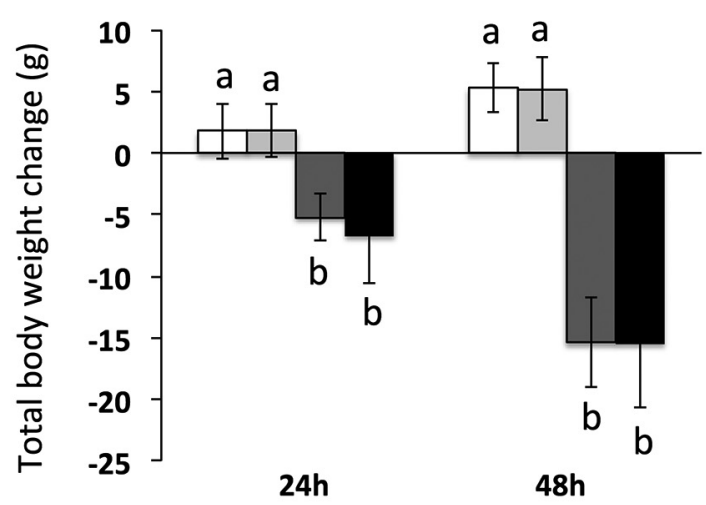

Figure 5. NTS $\rightarrow \mid P B N$ projections are required for cisplatin-induced anorexia and weight loss. $A$, Surgical setup for NTS $\rightarrow \mid P B N$ neuron inhibition: bilateral injection of CAV-2-Cre in the IPBN and the inhibitory Cre-dependent DREADD hM ${ }_{4}$ D in the caudal medial NTS. $B$, NTS $\rightarrow$ PBN neuron inhibition by CNO attenuated cisplatin-induced anorexia. C, NTS $\rightarrow$ PPBN neuron inhibition by CNO attenuated cisplatin-induced weight loss. D, Surgical setup for NTS $\rightarrow$ IPBN experiment in control rats: bilateral injection of CAV-2-Cre in the IPBN and AAV-mCherry in the NTS. $E$, CNO injection in control rats did not alter cisplatin-induced anorexia significantly. $F$, CNO injection in control rats did not alter cisplatin-induced weight loss significantly. Data are expressed as mean \pm SEM; different letters denote significant differences between groups within time points ( $p<0.05, \boldsymbol{B}$ and $\boldsymbol{C}: n=6-7$ per group, $\boldsymbol{E}$ and $\boldsymbol{F}: n=4-5 /$ group$)$.

way is glutamatergic. These data in rats are consistent with a very recent report identifying the glutamatergic phenotype of caudal NTS $\rightarrow$ IPBN axonal projections in mice, including synaptic inputs to IPBN CGRP neurons (Roman et al., 2016).

To test the functional relevance of the excitatory NTS $\rightarrow$ IPBN projection in mediating cisplatin-induced anorexia and body weight loss, we used an approach similar to that described above, but injected CAV-2-Cre into the IPBN and the inhibitory DREADD into the NTS. This approach permitted selective CNO-induced inhibition of NTS $\rightarrow$ IPBN neurons, which resulted in significant attenuation of cisplatin-induced anorexia and weight loss. Importantly, CNO had no effect on cisplatin-induced anorexia or body weight loss in rats expressing a control AAV. Therefore, the NTS $\rightarrow \mathrm{PBN}$ projection is necessary for anorexia and body weight loss after cisplatin treatment. 
Continued focus on defining the NTS neuron populations and mechanisms mediating cisplatin-induced anorexia will be important in future investigations. A logical starting point is to investigate neural populations known to be involved in energy balance control. For example, glucagon-like peptide-1 (GLP-1) is produced by caudal NTS neurons that innervate the IPBN (Alhadeff et al., 2014) and $~ 70 \%$ of IPBN GLP-1 fibers appose CGRP neurons closely (Richard et al., 2014). Central GLP-1 receptor signaling promotes anorexia, nausea, and vomiting (van Dijk and Thiele, 1999; Kinzig et al., 2003; Hayes et al., 2010; Kanoski et al., 2012). GLP-1 neurons are glutamatergic in rats (Zheng et al., 2015) and a subpopulation of NTS GLP-1 neurons are activated by cisplatin treatment (De Jonghe et al., 2016). Interestingly, central administration of a GLP-1 receptor antagonist attenuates but does not eliminate delayed-phase (i.e., $\geq 48 \mathrm{~h}$ after treatment) cisplatin-induced anorexia and pica (De Jonghe et al., 2016), the latter being a well established indicator of nausea/ malaise in rodents (Andrews and Horn, 2006). Because GLP-1 neurons are glutamatergic, blockade of GLP-1 receptors alone may be insufficient to block the effects of activating these neurons. In light of these collective findings, GLP-1 neurons may comprise a subpopulation of the NTS $\rightarrow$ IPBN neuron projection that our current study has demonstrated is required for cisplatininduced energy balance dysregulation. In addition, a recent report identified two discrete populations of cholecystokinin (CCK)- and dopamine $\beta$-hydroxylase (DBH)-expressing NTS neurons in which stimulation decreases food intake in mice (Roman et al., 2016). These two populations also innervate IPBN CGRP neurons directly in mice (Roman et al., 2016) and may also participate in the anorexic response to cisplatin treatment.

The current investigation used tools that permit chemogenetic inhibition of projection-specific neural populations using an approach similar to that which others have used in rats and mice (Carter et al., 2013; Boender et al., 2014). A limitation of this approach is that stereotaxic injections of viral vectors (both CAV2-Cre and the inhibitory AAV-DREADD) are unlikely to infect $100 \%$ of the target neurons of interest due to technical features of injection placement, virus spread, and neural uptake. For this reason, our behavioral results likely underestimate the actual role of $\mathrm{PBN} \rightarrow \mathrm{CeA}$ and NTS $\rightarrow$ IPBN projection neurons on cisplatininduced anorexia and body weight loss. In addition, it is possible that DREADDs were expressed in neurons in which CAV-2-Cre was transported from off-target sites; however, given that there are only very sparse anatomical projections from the caudal medial NTS and IPBN that target regions in close proximity to the IPBN or CeA, respectively (Norgren, 1978; Saper and Loewy, 1980; Herbert et al., 1990; Krukoff et al., 1992; Jhamandas et al., 1996), we find this possibility unlikely. A final limitation to our projection-specific approach is that it lacks neurochemical specificity. It will be important for future studies to investigate directly the functional significance of neurochemically identified neural populations (e.g., $1 \mathrm{PBN} \rightarrow$ CeA CGRP neurons; NTS $\rightarrow$ PBN GLP-1, CCK, and DBH neurons) in cisplatin-induced energy balance dysregulation.

Collectively, our results demonstrate that cisplatin's food intake and body weight suppressive actions depend on excitatory $\mathrm{PBNN} \rightarrow \mathrm{CeA}$ and NTS $\rightarrow \mathrm{PBN}$ projections. Our results highlight the direct functional role of hindbrain $\rightarrow$ forebrain projections in mediating cisplatin-induced side effects, providing neural circuit targets that can ultimately be leveraged for improved control of chemotherapy-induced anorexia and weight loss.

\section{References}

Alhadeff AL, Baird JP, Swick JC, Hayes MR, Grill HJ (2014) Glucagon-like peptide-1 receptor signaling in the lateral parabrachial nucleus contributes to the control of food intake and motivation to feed. Neuropsychopharmacology 39:2233-2243. CrossRef Medline

Alhadeff AL, Holland RA, Nelson A, Grill HJ, De Jonghe BC (2015) Glutamate receptors in the central nucleus of the amygdala mediate cisplatininduced malaise and energy balance dysregulation through direct hindbrain projections. J Neurosci 35:11094-11104. CrossRef Medline

Andrews PL, Horn CC (2006) Signals for nausea and emesis: Implications for models of upper gastrointestinal diseases. Auton Neurosci 125:100115. CrossRef Medline

Armbruster BN, Li X, Pausch MH, Herlitze S, Roth BL (2007) Evolving the lock to fit the key to create a family of $\mathrm{G}$ protein-coupled receptors potently activated by an inert ligand. Proc Natl Acad Sci U S A 104:51635168. CrossRef Medline

Boender AJ, de Jong JW, Boekhoudt L, Luijendijk MC, van der Plasse G, Adan RA (2014) Combined use of the canine adenovirus-2 and DREADD-technology to activate specific neural pathways in vivo. PLoS One 9:e95392. CrossRef Medline

Campos CA, Bowen AJ, Schwartz MW, Palmiter RD (2016) Parabrachial CGRP neurons control meal termination. Cell Metab 23:811-820. CrossRef Medline

Carter ME, Soden ME, Zweifel LS, Palmiter RD (2013) Genetic identification of a neural circuit that suppresses appetite. Nature 503:111-114. CrossRef Medline

Carter ME, Han S, Palmiter RD (2015) Parabrachial calcitonin gene-related peptide neurons mediate conditioned taste aversion. J Neurosci 35:45824586. CrossRef Medline

Cohen L, de Moor CA, Eisenberg P, Ming EE, Hu H (2007) Chemotherapyinduced nausea and vomiting: incidence and impact on patient quality of life at community oncology settings. Support Care Cancer 15:497-503. CrossRef Medline

De Jonghe BC, Horn CC (2008) Chemotherapy-induced pica and anorexia are reduced by common hepatic branch vagotomy in the rat. Am J Physiol Regul Integr Comp Physiol 294:R756-R765. CrossRef Medline

De Jonghe BC, Horn CC (2009) Chemotherapy agent cisplatin induces $48 \mathrm{~h}$ Fos expression in the brain of a vomiting species, the house musk shrew (Suncus murinus). Am J Physiol Regul Integr Comp Physiol 296:R902R911. CrossRef Medline

De Jonghe BC, Holland RA, Olivos DR, Rupprecht LE, Kanoski SE, Hayes MR (2016) Hindbrain GLP-1 receptor mediation of cisplatin-induced anorexia and nausea. Physiol Behav 153:109-114. CrossRef Medline

Grunberg SM (2012) Chemotherapy-induced nausea and vomiting incidence and prevalence. Am Soc Clin Oncol Educ Book 541-543.

Hattori T, Yakabi K, Takeda H (2013) Cisplatin-induced anorexia and ghrelin. Vitam Horm 92:301-317. CrossRef Medline

Hayes MR, De Jonghe BC, Kanoski SE (2010) Role of the glucagon-likepeptide-1 receptor in the control of energy balance. Physiol Behav 100: 503-510. CrossRef Medline

Herbert H, Moga MM, Saper CB (1990) Connections of the parabrachial nucleus with the nucleus of the solitary tract and the medullary reticular formation in the rat. J Comp Neurol 293:540-580. CrossRef Medline

Hesketh PJ (2008) Chemotherapy-induced nausea and vomiting. N Engl J Med 358:2482-2494. CrossRef Medline

Hesketh PJ, Bohlke K, Lyman GH, Basch E, Chesney M, Clark-Snow RA, Danso MA, Jordan K, Somerfield MR, Kris MG; American Society of Clinical Oncology (2016) Antiemetics: American Society of Clinical Oncology Focused Guideline Update. J Clin Oncol 34:381-386. CrossRef Medline

Hong YS, Lee HR, Park S, Lee SC, Hwang IG, Park BB, Lee J, Ahn JS, Ahn MJ, Lim HY, Park K (2006) Three-week schedule of irinotecan plus cisplatin in patients with previously untreated extensive-stage small-cell lung cancer. Br J Cancer 95:1648-1652. CrossRef Medline

Horn CC (2009) Brain Fos expression induced by the chemotherapy agent cisplatin in the rat is partially dependent on an intact abdominal vagus. Auton Neurosci 148:76-82. CrossRef Medline

Horn CC, Ciucci M, Chaudhury A (2007) Brain Fos expression during $48 \mathrm{~h}$ after cisplatin treatment: neural pathways for acute and delayed visceral sickness. Auton Neurosci 132:44-51. CrossRef Medline

Jhamandas JH, Petrov T, Harris KH, Vu T, Krukoff TL (1996) Parabrachial nucleus projection to the amygdala in the rat: electrophysiological and anatomical observations. Brain Res Bull 39:115-126. CrossRef Medline 
Jordan K, Jahn F, Aapro M (2015) Recent developments in the prevention of chemotherapy-induced nausea and vomiting (CINV): a comprehensive review. Ann Oncol 26:1081-1090. CrossRef Medline

Kanoski SE, Rupprecht LE, Fortin SM, De Jonghe BC, Hayes MR (2012) The role of nausea in food intake and body weight suppression by peripheral GLP-1 receptor agonists, exendin-4 and liraglutide. Neuropharmacology 62:1916-1927. CrossRef Medline

Kinzig KP, D’Alessio DA, Herman JP, Sakai RR, Vahl TP, Figueiredo HF, Murphy EK, Seeley RJ, Figueredo HF (2003) CNS glucagon-like peptide-1 receptors mediate endocrine and anxiety responses to interoceptive and psychogenic stressors. J Neurosci 23:6163-6170. Medline

Krukoff TL, Morton TL, Harris KH, Jhamandas JH (1992) Expression of c-fos protein in rat brain elicited by electrical stimulation of the pontine parabrachial nucleus. J Neurosci 12:3582-3590. Medline

McLean IW, Nakane PK (1974) Periodate-lysine-paraformaldehyde fixative: a new fixation for immunoelectron microscopy. J Histochem Cytochem 22:1077-1083. CrossRef Medline

Norgren R (1978) Projections from the nucleus of the solitary tract in the rat. Neuroscience 3:207-218. CrossRef Medline

Paxinos G, Watson, C (2005) The rat brain in stereotaxic coordinates, Ed 5. San Diego: Academic.

Percie du Sert N, Rudd JA, Apfel CC, Andrews PL (2011) Cisplatin-induced emesis: systematic review and meta-analysis of the ferret model and the effects of 5-HT(3) receptor antagonists. Cancer Chemother Pharmacol 67:667-686. CrossRef Medline
Richard JE, Farkas I, Anesten F, Anderberg RH, Dickson SL, Gribble FM, Reimann F, Jansson JO, Liposits Z, Skibicka KP (2014) GLP-1 receptor stimulation of the lateral parabrachial nucleus reduces food intake: neuroanatomical, electrophysiological and behavioral evidence. Endocrinology 155:4356-4367. CrossRef Medline

Roman CW, Derkach VA, Palmiter RD (2016) Genetically and functionally defined NTS to PBN brain circuits mediating anorexia. Nat Commun 7:11905. CrossRef Medline

Rudd JA, Andrews PLR (2005) In: Management of nausea and vomiting in cancer and cancer treatment (Hesketh PJ, ed), pp 15-65. Sudbury, MA: Jones and Bartlett.

Saper CB, Loewy AD (1980) Efferent connections of the parabrachial nucleus in the rat. Brain Res 197:291-317. CrossRef Medline

Schwaber JS, Sternini C, Brecha NC, Rogers WT, Card JP (1988) Neurons containing calcitonin gene-related peptide in the parabrachial nucleus project to the central nucleus of the amygdala. J Comp Neurol 270:416426, 398-399. Medline

van Dijk G, Thiele TE (1999) Glucagon-like peptide-1 (7-36) amide: a central regulator of satiety and interoceptive stress. Neuropeptides 33:406414. CrossRef Medline

Winton T, et al. (2005) Vinorelbine plus cisplatin vs observation in resected non-small-cell lung cancer. N Engl J Med 352:2589-2597. CrossRef Medline

Zheng H, Stornetta RL, Agassandian K, Rinaman L (2015) Glutamatergic phenotype of glucagon-like peptide 1 neurons in the caudal nucleus of the solitary tract in rats. Brain Struct Funct 220:3011-3022. CrossRef Medline 